\title{
Water as a geoecologic product and ultimate shared resource: towards sustainability
}

\begin{abstract}
Water is the agent that links the global hydrologic cycle to ecosystems, humans, and civilization. The hydrologic cycle integrates landscapes, biophysical and sociocultural processes. Water allocation criteria must be expanded to insure ecological water flows to watersheds, wetlands and estuarine systems. Historically water has been perceived as a common good, that once shared was able to build great and longlasting civilizations. The combined perspective of landscape, society and nature provides numerous opportunities for studying examples of constructive cooperation grounded on shared values and designs with nature, both in terms of structure as well as processes to establish the foundation for sustainable management of water in the ecological commons. Water management has been a critical factor in the emergence, development, and endurance of the first civilizations. Local stakeholders can exert effective governance that is flexible and capable of quick change detection and adaptation. This flexibility is not easily achieved by hard laws and regulations that are generally inflexible, too general and lack local relevance. Here we highlight that water resource management must adopt a resilience-based approach to create long lasting partnerships with nature grounded in an ecosystem-based approach, maximizing beneficial outcomes while reducing control by rigid regulation.
\end{abstract}

Keywords: geoecosystems sustainability, water management, water sharing cultures, humans and natural processes, water as a common good, shared stewardship
Volume 2 Issue I - 2018

\author{
Yara Schaeffer-Novelli, ${ }^{1,2}$ Gilberto Cintrón- \\ Molero \\ 'Department of Biological Oceanography, University of São \\ Paulo, Brazil \\ ${ }^{2}$ Instituto Bioma Brasil, Brazil
}

\begin{abstract}
Correspondence: Yara Schaeffer-Novelli, Department of Biological Oceanography, Oceanographic Institute, University of São Paulo, São Paulo, Brazil,Tel +55I 1999978402 ,
\end{abstract} Email novelliy@usp.br

Received: January 14, 2018 | Published: February 21, 2018

\section{Opinion}

Water is life and is the agent that links the global hydrologic cycle to ecosystems, humans, and civilization. The hydrologic cycle integrates humans with the planet's life sustaining system regulating the presence of life; its, development and eventual persistence. Thus, water management has been a critical factor in the emergence, development, and endurance of the first civilizations. Water allocation issues can lead to conflict or peace. However, can be a catalyst for cooperation if wisely managed. Water allocation remains a critical topic and, an increasingly important topic when the full scope of water needs becomes recognized. Not only is water important for societal needs; it is critical for ecosystems that support society. Furthermore, water vapor is actually the planets dominant greenhouse gas and latent heat release drives circulation stability, weather and climate. The hydrologic cycle integrates landscapes, biophysical and sociocultural processes. Thus, water allocation criteria must be expanded to insure ecological water flows to watersheds, wetlands and estuarine systems. The alterations in environmental flows have severe consequences on coastal systems, reducing sedimentation, increasing subsidence and allowing increases in coastal erosion and retreat at a time of predicted sea level rise. The indirect consequences of the unrestricted expansion of economic activity at any cost in fluvial systems, detached from appropriate environmental concerns is undermining the planet's life sustaining capacity decreasing the performance in the delivery of many ecosystem services and, worse decreasing future options for regenerative capacity and to address future needs. We suggest that water management must adopt a broader multiscale dynamic problem framing approach able to accommodate to varying spatial and temporal scales. Such dynamic framing recognizes natures' hierarchical organization and the fact that complex problems cannot be managed based on small spatial and short temporal scales. Many environmental problems emerge from the incoherence between the generally limited human planning horizon and nature's activity domain. Here we highlight that water resource management must adopt a resilience-based approach. The resilience of a system is inversely proportional to the magnitude of a stressor and directly proportional to the gradualism of the recovery rate. Furthermore, resilience is scale dependent and scales are interdependent. The landscape, as a flexible level of observation is well suited for dealing with nature's panarchial organization ${ }^{1}$ provides a suite of focal levels capable of framing problems at the most relevant spatiotemporal domains as well as a framework to assess cross scale interactions. However this perspective is still not widely understood by many decision makers. In ecology it is now widely recognized that a triadic approach is required for explaining reality. ${ }^{2}$ First the system is carefully defined at a relevant level of observation, called the focal level, then immediately at levels above and below; these levels provide constraints and mechanisms. The landscape perspective is particularly important because it integrates human and natural processes allowing spatial planning to capture the combined dynamics and synergies of social ecological that shape an emergent total geoecological system. The combined perspective of landscape, society and nature provides several opportunities for studying examples of constructive cooperation grounded on shared values and designing with nature, both in terms of structure as well as processes to strengthen the foundation for sustainable management of water in the ecological commons. However, the ecological commons we consider here is one that considers social, economic and cultural influences le degradation. This is not Hardin's, ${ }^{3}$ unmanaged, unregulated commons where unrestricted exploitation for a finite resource leads to its inexorable degradation or depletion. Such degradation is not inevitable, it can be 
arrested by integrating human cognition and activity coherently with biophysical processes. Because such a combined system involves knowledge and experience it has been referred to as a sustainable nöösystem perspective. ${ }^{4}$

Water, however, can be a source of conflict when make scarce by greed, waste, or perceiving it as a commodity rather as a common good (a geoecological good) to be shared. However, historically water has been perceived as a common good, that once shared was able to build the great and long-lasting civilizations. Although these societies were often associated with great river systems, others developed even in water scarce regions, where agriculture depended on sophisticated irrigation systems. Thus, even where limited, cultures self-organized to develop sharing networks; aqueducts or asequia networks; these community ditches are ancient landscape-scale features associated with water sharing cultures. The term asequia is derived from the Arabic language and the technology was brought to Iberia during their occupation and was adapted by the Portuguese (in the form of the levadas still foundin Madeira Island). In the US asequias were stablished more than four hundred years ago by Spanish settlers and continue to provide water for farming in areas of the US once occupied by Spain. The asequia culture is associated with the unique principle of water sharing; the asequia system is grounded in a form of water democracy. Another notable example of self-organization to create water sharing cultures is the Turpan water system (locally known as karez or wells in the local Uygür language). This water system was crucial in the development of Turpan an important stopover in the Silk Road. Turpan, located in the vicinity of Taklimakan desert owed its development to the karez system. Wells are dug tap groundwater and then networked by a complex gallery system. The Chinese karez are over 2,000 years old. The quanats of Gonabad (Iran) date from $700 \mathrm{BC}$ and are still used today. These systems are not only a global heritage of human civilization but they are good examples of adaptive governance, and water democracy coherent with natural processes, sustainability, and water sharing. These shared water systems reflect millennia of cultural knowledge that links human culture and ecology for sustainability. They reflect the self-organization of a social ecological system which is well coupled to natural processes and synergies that shape what is essentially a geoecological system. Such self-organization is an emergent property of complex systems and is examples of adaptive governance coherent with natural processes. These water sharing systems are robust as demonstrated by their endurance. This robustness allowed user communities to persist in extremely harsh environments while attaining high levels of social and economic and development through shared partnership with nature and successful stewardship. Here we suggest that one of the greatest obstacles contemporary society faces is an issue of environmental literacy; ${ }^{1}$ reflected in its tenacious tendency to think in simplistic terms of a single equilibrium state and an infinitely resilient nature that inexorably returns to equilibrium after being stressed. This mindset has been reinforced by thinking in terms of small spatial and short temporal scales while neglecting the existence of multiple stability domains; that is neglecting cross-scale dynamics whereas the hydrologic cycle is a global scale feature that acts at broad spatial and temporal scales involving cross-scale interactions as well as long term cycles of variable behavior. Many decision makers are not familiar with hierarchic organization, complex systems, resilience and regime shifts. As a result, misleading arguments arise grounded in false premises such as reductionism vs holism when in the context of complex systems both approaches are complementary and "nonagonistic" apparent conflict at one scale can become mutualism at a higher level.

What is critical in the context of water resources management for sustainability is the urgent need for developing new problem framing perspectives that are dynamic; able to frame issues at multiple scales in order to capture issues spanning small and large scales as well as cross-scale interactions. A single scale perspective is not adequate to capture the dynamics or the complexity of the geoecological process that influence water availability and water allocation issues. It must be recognized that water is not a mere ecological service; it is part of an intricate web of functions that configure high-level meta-services that support systemic self-organization at broad scale. These metaservices cannot be unbundled, so management must necessarily broaden its managerial domain to at least local landscape or regional landscape assemblage scales. Ecological robustness depends on spatial resilience. Landscapes are composed of mosaics of locally adapted units with the capacity for regeneration and reorganization as required to address disturbances and change at varying scales. High diversity entails high capacity for adaptation and coupling. Essentially this heterogeneity reflects nature's "All weather investment strategy". Investment here is used in terms of the "currency used by landscapes for regeneration, exploration and adaptation" (that is, number of species). One of the principal emergent in ecological functions of landscapes is evolvability; evolutionary work. This evolvability is the system's "learning capacity" or "strength in reserve" stored as "overhead"; 5 the capacity for adaptive evolution. One could say that landscape persistence is based on strategic tinkering that allows a geoecological system to reconfigure itself when ecological conditions become untenable. The broad scale perspective entails the notion of the ecological commons as a geoecological system; not as the open access unregulated space of the "Tragedy of the Commons". ${ }^{3}$ Rather, we refer to the ecological commons as a life support system. In this ecological commons "Common Property Protocols" offer the means to protect and allocate resources through community norms that regulate the amount, timing and tools used to extract resources. These norms are often closely linked to natural processes and shape adaptive governance systems coherent with natural processes and shape adaptive governance systems which are highly coherent with natural processes. Thus, we suggest that scientists support decision makers in helping understand the need for water and llandscape level management must be grounded in the following:

a. Adopt spatial planning approached incorporating dynamic spatiotemporal framing, capable of being shifted to capture both the big picture (scope) and detail (grain) as systems develop or evolve,

b. Incorporate means to promote situational awareness and preemptive (feed-forward) that provides for strategic hedging based on avoidance and augmenting resilience,

c. Encourage Participatory dialogical process grounded in shared interests, alliances and partnerships that integrate different perspectives,

d. Endorse learning and education based where stakeholders share formal and tacit knowledge as well as scientific knowledge and local expectations to create shared destinies,

e. Base project/activity planning and design on management for resilience, nimbleness/self-organization, 
f. Focusing attention only at one level is not enough; a macroscopic view requires a triadic perspective involving focal level and lower level dynamics, as well as higher level context and its long-term integrity.

\section{Conclusion}

The power of cognition allows humans to derive lessons and knowledge from intimacy with place. In local communities this is embedded in tacit, traditional knowledge, local wisdom which is a component of system-level self-organization. The outcome of such virtuous or skillful governance has the potential for long-term multigenerational management grounded in local knowledge, caring, sharing and responsibility that shape partnership with nature. Policy makers must adopt a broader, dynamic perspective for insuring the sustainability of water resources. As a product and component of a global earth surface system water is a shared resource and is best managed by means of collaborative processes coupled with natural processes. Complex problems are best addressed taking advantage of natural subsidies; local processes and self-organization. Selforganization is facilitated by broad scale processes as well as local resilience. The landscape level of observation is the most appropriate to capture the interaction and fusing of ecologic and social, cultural and economic system into a single geoecosystem. The so called "commons" are in fact constitutive components of that geoecosystem; and are best managed by entities that are familiar with local system properties, dynamics and behavior. Because of their traditional knowledge and close interdependence these stakeholders can exert effective governance that is flexible and capable of quick change detection and adaptation. This flexibility is not easily achieved by hard laws and regulations that are generally inflexible, too general and lack local relevance.

\section{Acknowledgements}

The authors acknowledge the team at Intituto BiomaBrasil-IBB, a nonprofit organization concerned with the use of science to ensure social-ecological sustainability.

\section{Conflict of interest}

Authors declare there is no conflict of interest in publishing the article.

\section{References}

1. Holing CS. The resilience of terrestrial ecosystems: Local surprise and global change. In: Clark WC \& Munn RE, editors. Sustainable Development of the Biosphere, Cambridge University Press, UK; 1986. p. 292-317.

2. Saithe SN. Evolving Hierarchical Systems: Their Structure and Representation. Columbia University Press, USA; 1985. p. 1-343.

3. Hardin G. The tragedy of the commons. Science New Series. 1968;162(3859):1243-1248.

4. Barret GW, Peles JD, Odum EP. Transcending processes and the levelsof-organization concept. BioScience. 1977;47(8):531-535.

5. Ulanowicz RE. Ecology the ascendent perspective, 1st Edn. Columbia University Press, USA; 1997. p. 1-201. 